\title{
Penile revascularization has good long-term success rates
}

Although considered technically

challenging, the authors of a new study suggest that penile revascularization surgery is a viable treatment option for men with vasculogenic erectile dysfunction (ED). Phosphodiesterase type 5 (PDE5) inhibitors are the current mainstay of ED therapy, but low rates of compliance and high rates of failure leave the field open to alternatives.

Onder Kayıgil and colleagues at the Clinic of Ankara Ataturk Training and Research Hospital in Turkey have reported the second largest series of revascularization operations to date, involving 110 men, who completed a mean of 73.2 months follow-up duration.

The principle of penile revascularization —also known as microvascular arterial bypass surgery-is to restore blood flow to the cavernosal body. Numerous operative techniques have been described with varying success rates. Here, the surgeons created an end-to-side anastomosis between the inferior epigastric artery (IEA) and the deep dorsal vein. Other techniques include end-to-end anastomosis of the IEA to the dorsal artery, direct anastomosis to cavernosal bodies or cavernosal artery, and triple anastomoses between the dorsal penile vein, dorsal penile artery and the IEA.

\section{4 ...revascularization might} gain popularity in the future as a third-line treatment option... 77

Patients were assessed before and after surgery using the International Index of Erectile Function-5 (IIEF-5) questionnaire, with a 5-point increase in score following surgery considered a success. Mean IIEF-5 score increased from 7.3 before the operation to 16.8 at the end of follow-up, with a success rate of $72.7 \%$. Minor complication rates were low, although seven patients experienced the major complication of glans hypervascularization.

Patient selection for surgery is crucial; penile color-flow Doppler ultrasonography was used to identify patients with arterial insufficiency or veno-occlusive dysfunction, the most likely to benefit from revascularization. Researchers also report the use of preoperative corpus cavernosum electromyography (CC-EMG) to assess changes in cavernosal electrical activity and exclude men with patterns suggestive of neurogenic ED. CC-EMG might also be used to measure the cavernosal smooth muscle tone.

The authors conclude by articulating their hope that revascularization might gain popularity in the future as a third-line treatment option, after failure of PDE5 inhibitors and intracavernosal drug injections.

Sarah Payton

Original article Kayıgil, 0. et al. Penile revascularization in vasculogenic erectile dysfunction (ED): long-term follow-up. BJU Int. doi:10.1111/j.1464-410X.2011.10293.x 